\title{
The Effect of Ageing on In Vivo Human Ciliary Muscle Morphology and Contractility
}

\author{
Amy L. Sheppard and Leon N. Davies
}

Purpose. To assess the effect of ageing on in vivo human ciliary
muscle morphology and contractility during accommodation.

Methods. Seventy-nine subjects, aged 19-70 years were recruited. High-resolution images were acquired of nasal and temporal ciliary muscle in the relaxed state, and at stimulus vergence levels of -4 and $-8 \mathrm{D}$, using anterior segment optical coherence tomography (AS-OCT). Objective refractions and axial lengths were also recorded. Linear regression analysis was performed to determine the effect of age on nasal and temporal ciliary muscle morphologic characteristics.

Results. Ciliary muscle anterior length decreased significantly with age both nasally $(R=0.461, P=0.001)$ and temporally $(R=0.619, P<0.001)$ in emmetropic eyes. In a subset of 37 participants, ciliary muscle maximum width increased significantly with age, by $2.8 \mu \mathrm{m} /$ year nasally $(R=0.54, P<0.001)$ and $3.0 \mu \mathrm{m} /$ year temporally $(R=0.44, P=0.007)$, while the distance from the inner apex of the ciliary muscle to the scleral spur decreased significantly with age on both the nasal and temporal aspects $(R=0.47 ; P=0.004$ and $R=0.43 ; P=$ 0.009 , respectively). During accommodation, changes to ciliary muscle thickness and length remained constant throughout life.

Conclusions. The human ciliary muscle undergoes age-dependent changes in morphology that suggest an antero-inwards displacement of muscle mass, particularly in emmetropic eyes. However, the morphologic changes observed appear not to affect the ability of the muscle to contract during accommodation, even in established presbyopes, thus supporting a lenticular model of presbyopia development. (Invest Ophthalmol Vis Sci. 2011;52:1809-1816) DOI:10.1167/iovs.10-6447

W hile it is generally accepted that changes to the size and mechanical properties of the crystalline lens are central to the development of presbyopia, ${ }^{1-5}$ changes in other associated structures, including the ciliary muscle, ${ }^{6}$ choroid, ${ }^{7,8}$ and vitreous body ${ }^{9}$ are also likely to have a contributory effect. Age-related changes in ciliary muscle have been documented previously, from a range of primate studies and in vitro investigations. In vivo data describing the effects of age on human ciliary muscle morphology and function are scarce, ${ }^{1}$ as the iris prevents direct visualization of the ciliary region. ${ }^{10,11}$ Post mortem microscopic examination of human ciliary body tissue

From the Ophthalmic Research Group, Life and Health Sciences, Aston University, Birmingham, United Kingdom.

Supported by a PhD Scholarship from Bausch \& Lomb, USA (ALS) and a College of Optometrists Research Fellowship Award, UK (LND).

Submitted for publication August 20, 2010; revised October 7, 2010; accepted October 7, 2010.

Disclosure: A.L. Sheppard, Bausch \& Lomb (F); L.N. Davies, None

Corresponding author: Leon N. Davies, Ophthalmic Research Group, Aston University, Birmingham, UK B4 7ET;

1.n.davies@aston.ac.uk. has revealed that the ciliary muscle decreases in both length and area with age. ${ }^{6,12}$ Pardue and Sivak $^{6}$ found that anterior ciliary muscle length (measured perpendicular from the line of maximum muscle thickness to the scleral spur) showed a greater reduction with age than overall length measures: in a relatively small sample of 16 pairs of eyes, mean anterior length was approximately $680 \mu \mathrm{m}$ in donors aged under 34 years, and $550 \mu \mathrm{m}$ in older eyes aged up to 107 years. Furthermore, the trend for maximum muscle thickness to increase over time, ${ }^{6}$ while the distance from the scleral spur to the inner apex of the ciliary muscle decreases, ${ }^{12}$ indicate that the human ciliary muscle adopts a more anterior-inward position with advancing age. In vivo MRI data have shown an age-related reduction in ciliary muscle ring diameter, ${ }^{13,14}$ which could be due to ciliary body thickening, and a decrease in axial distance from cornea to muscle apex, ${ }^{11}$ representing an anterior shift of the ciliary body.

In the primate eye, age-related nasal/ temporal biometric asymmetry develops in the ciliary region. In vivo ultrasound biomicroscopic examination of rhesus monkey eyes has identified a more significant reduction in temporal, compared to nasal, circumlental space (CLS) with age. ${ }^{15,16}$ Additionally, age and temporal CLS (but not nasal CLS) are together a better indicator of accommodative amplitude than age alone, ${ }^{16}$ suggesting that some process linked to presbyopia development may predominantly impact the temporal aspect of the eye. No such age-related asymmetry has been detected in the human eye using either in vitro or in vivo methods, although previous authors have generally not clarified the aspect of ciliary muscle investigated (i.e., nasal/ temporal/ superior/ inferior). ${ }^{6,12,14} \mathrm{We}$ recently reported significantly greater ciliary muscle thickness and contractile response (as inferred by reduced anterior length), on the temporal side, compared to the nasal aspect in young eyes. ${ }^{17}$ These observed differences in both morphology and contraction highlight the need to consider nasal and temporal ciliary muscle regions separately.

The effects of age on human ciliary muscle contractility are contentious, with some contradictory findings in previously published studies. Human ciliary muscle has been shown in vitro to maintain its contractile response to pilocarpine administration, demonstrating a reduction in length and maximum width, even in eyes with advanced presbyopia. ${ }^{6}$ However, in vitro studies alone are insufficient to confirm the contractile changes in ciliary muscle with age. ${ }^{13}$ Post mortem tissue changes and ischemic effects could alter the response of the muscle to topically applied pharmacologic agents. Furthermore, samples from sectioned eyes may not accurately reflect the in vivo responses of human ciliary muscle.

Hitherto, high resolution MRI ${ }^{11,13,14}$ and ultrasound biomicroscopy (UBM) ${ }^{10}$ have been used to analyze in vivo the contractility of ageing ciliary muscle. Park et al. ${ }^{10}$ observed minimal inwards ciliary body movement after pilocarpine administration in a cohort of 15 presbyopic subjects (mean age 65.3 years) before cataract surgery. Post-operatively, pharmacologically induced centripetal movement significantly increased, suggest- 


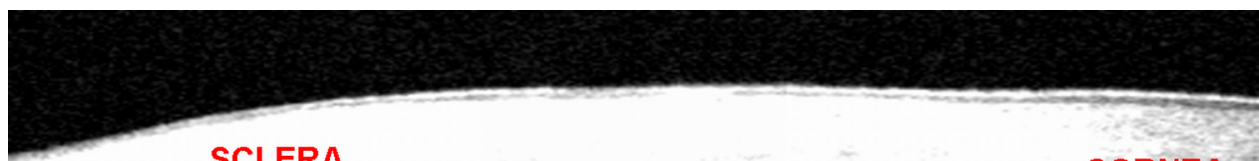

SCLERA

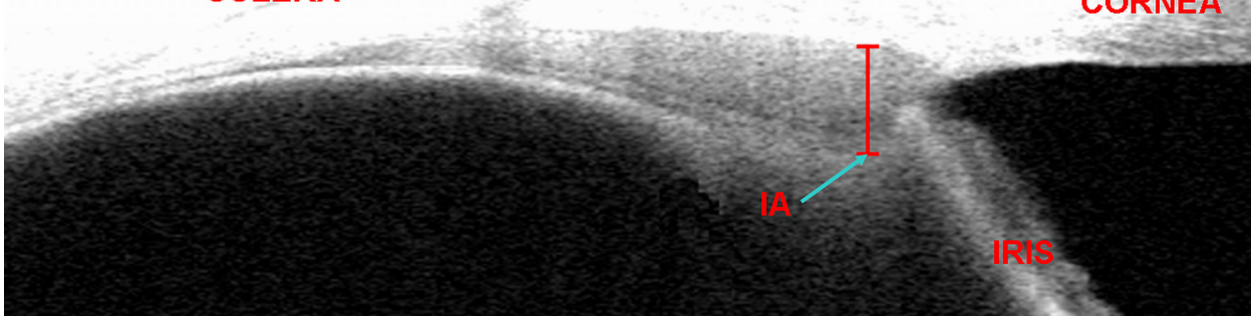

FigurE 1. Measurement of temporal ciliary muscle maximum thickness. A caliper positioned at the inner apex (IA) is extended to the ciliary muscle/ sclera interface. Here, maximum thickness $=0.84 \mathrm{~mm}$. ing that lenticular sclerotic changes may hinder ciliary muscle contractility in older eyes, due to altered anterior segment geometry. In contrast to these findings, published MRI data indicate that the inwards contractile movement of the ciliary muscle is undiminished by age, ${ }^{13,14}$ and not affected by intraocular lens (IOL) implantation. ${ }^{14}$ Human ciliary muscle ring diameter decreases by approximately $0.025 \mathrm{~mm}$ per year, but the mean reduction of $0.64 \pm 0.05 \mathrm{~mm}$ in this parameter in response to an $8 \mathrm{D}$ stimulus was found to be independent of subject age. In pseudophakic eyes, the contractile change in ciliary muscle ring diameter was statistically identical with contralateral phakic eyes. ${ }^{14}$ A further, recently-published, MRI study analyzing ciliary muscle action in vivo identified an ageindependent increase in ciliary muscle thickness with accommodation, in volunteers aged from 22-91 years. ${ }^{11}$ However, no anterior contractile movement of the muscle apex was observed in any of the 32 phakic subjects, challenging the generally-accepted model of accommodation, in which a concurrent forwards and inwards shift of ciliary muscle mass acts to reduce tension on the zonules. ${ }^{2,18,19}$

The majority of available evidence, from both in vitro and in vivo studies, therefore, indicates that human ciliary muscle maintains its contractile ability long after the onset of presbyopia, although whether the nature of the response varies with age is less obvious. Pilocarpine is known to cause powerful ciliary muscle contraction and act as a superstimulus to accommodation, ${ }^{20-22}$ so the use of such pharmacologic agents should ideally be avoided to analyze natural ciliary muscle function in vivo. Our recent data provide support for an anterior, as well as centripetal, shift of ciliary muscle mass during stimulus-driven accommodation, ${ }^{17}$ but whether this pattern of response persists in older eyes is unknown.

Regarding ciliary muscle morphology, age-related reductions in length (particularly of the anterior portion) and area have been documented in vitro, but not yet confirmed in vivo. In vitro and MRI data indicate that the human ciliary muscle increases in maximum width throughout life, and slowly adopts a more anterior-inward position. Possible nasal versus temporal variations in age-dependent morphologic changes have not previously been investigated in the human eye, although asymmetry has been shown to develop in the monkey ciliary region. ${ }^{15,16}$ There is a lack of published in vivo data collected using the same high-resolution methodology across a broad range of age groups documenting the effects of age on human ciliary muscle morphology and contractility. Anterior segment optical coherence tomography (AS-OCT) has been shown to be a valid tool for analysis of ciliary muscle biometry, ${ }^{17,23}$ with an axial resolution of up to $8 \mu \mathrm{m},{ }^{24}$ which is superior to the values of $0.156 \mathrm{~mm},{ }^{13,14}$ and more recently, $0.078 \mathrm{~mm},{ }^{11}$ reported in previous MRI-based studies.

The aim of this study, therefore, is to provide new highresolution in vivo data on the age-related changes in human ciliary muscle morphology, using AS-OCT. The possible development of nasal versus temporal asymmetry in these changes will be explored, with a consideration of the implication on presbyopia development. Furthermore, the accommodative responses of ageing ciliary muscle will be compared to those of young eyes to attempt to clarify the effects of age on contractility.

\section{Methods}

\section{Subjects}

A cohort aged 35 years and older was required for the study, to investigate ciliary muscle characteristics from incipient presbyopia onwards. The data acquired from this sample were compared with a young cohort described previously, ${ }^{17}$ aged $19-34$ years (mean $25.8 \pm$ 4.5 years). Twenty-nine older volunteers aged 35-70 years, with no history of ocular abnormality or intraocular surgery were recruited for the study, using email announcements at Aston University. The mean age of older participants was $46.3 \pm 10.2$ years. The younger and older cohorts together comprised 79 subjects aged 19-70 years (mean $33.3 \pm 12.2$ years). The complete sample, therefore, represented

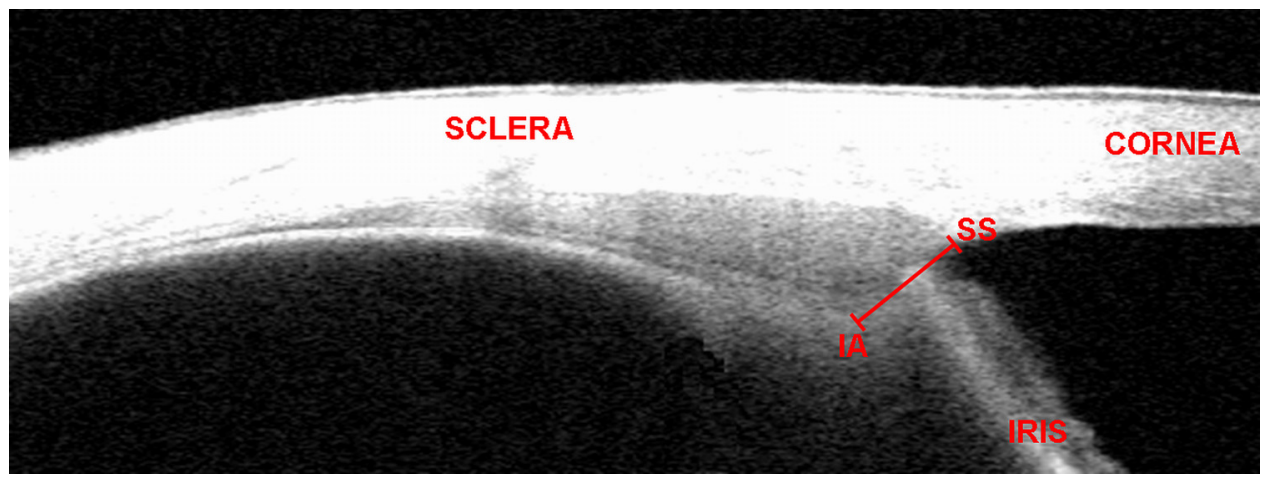

FIGURE 2. Measurement of distance from inner apex (IA) to scleral spur (SS) on the temporal side. Here, distance from inner apex to scleral spur $=0.92 \mathrm{~mm}$. 
TABLE 1. Change in Ciliary Muscle Morphologic Characteristics with Age

\begin{tabular}{|c|c|c|c|}
\hline \multirow{2}{*}{$\begin{array}{l}\text { Ciliary Muscle } \\
\text { Parameter }\end{array}$} & \multicolumn{3}{|c|}{ Change with Age } \\
\hline & Side & Emmetropes & Myopes \\
\hline \multirow[t]{2}{*}{ Total length } & Nasal & No significant change; Mean $=4.53 \pm 0.57 \mathrm{~mm}$ & No significant change; Mean $=4.84 \pm 0.65 \mathrm{~mm}$ \\
\hline & Temporal & No significant change; Mean $=4.69 \pm 0.66 \mathrm{~mm}$ & No significant change; Mean $=5.07 \pm 0.77 \mathrm{~mm}$ \\
\hline \multirow[t]{2}{*}{ Anterior length } & Nasal & $\begin{array}{l}\text { Decreases: Nasal AL }(\mathrm{mm})=0.989(0.00546 * \text { age }) \\
\quad R=0.461, P=0.001\end{array}$ & No significant change; Mean $=0.91 \pm 0.15 \mathrm{~mm}$ \\
\hline & Temporal & $\begin{array}{l}\text { Decreases: Temporal AL }(\mathrm{mm})=1.080(0.00895 * \text { age }) \\
\quad R=0.619, P<0.001\end{array}$ & No significant change; Mean $=0.92 \pm 0.15 \mathrm{~mm}$ \\
\hline \multirow[t]{2}{*}{ CM25 } & Nasal & No significant change; Mean $=528 \pm 44 \mu \mathrm{m}$ & No significant change; Mean $=541 \pm 62 \mu \mathrm{m}$ \\
\hline & Temporal & No significant change; Mean $=541 \pm 50 \mu \mathrm{m}$ & No significant change; Mean $=546 \pm 66 \mu \mathrm{m}$ \\
\hline \multirow[t]{2}{*}{ CM50 } & Nasal & No significant change; Mean $=284 \pm 42 \mu \mathrm{m}$ & No significant change; Mean $=304 \pm 43 \mu \mathrm{m}$ \\
\hline & Temporal & $\begin{array}{l}\text { Decreases: Temporal CM50 }(\mu \mathrm{m})=383(1.940 * \text { age }) \\
\quad R=0.448, P=0.002\end{array}$ & $\begin{array}{l}\text { Decreases: Temporal CM50 }(\mu \mathrm{m})=581(3.810 * \text { age }) \\
\quad R=0.538, P=0.001\end{array}$ \\
\hline \multirow{2}{*}{$\mathrm{CM} 75$} & Nasal & No significant change; Mean $=143 \pm 22 \mu \mathrm{m}$ & No significant change; Mean $=157 \pm 22 \mu \mathrm{m}$ \\
\hline & Temporal & $\begin{array}{l}\text { Decreases: Temporal CM75 }(\mu \mathrm{m})=184(0.760 * \text { age }) \\
\quad R=0.380, P=0.010\end{array}$ & $\begin{array}{l}\text { Decreases: Temporal CM50 }(\mu \mathrm{m})=276(1.26 * \text { age }) \\
\quad R=0.410, P=0.016\end{array}$ \\
\hline \multirow[t]{2}{*}{ CM2 } & Nasal & No significant change; Mean $=327 \pm 59 \mu \mathrm{m}$ & No significant change; Mean $=372 \pm 72 \mu \mathrm{m}$ \\
\hline & Temporal & $\begin{array}{l}\text { Decreases: Temporal CM2 }(\mu \mathrm{m})=434(2,079 * \text { age }) \\
\quad R=0.387, P=0.009\end{array}$ & No significant change; Mean $=419 \pm 74 \mu \mathrm{m}$ \\
\hline
\end{tabular}

$n=45$ emmetropes/hyperopes (MSE $=-0.75$ to $+2.33 \mathrm{D}$ ), mean age, $34.7 \pm 12.8$ years; and 34 myopes $($ MSE $<-0.75 \mathrm{D})$, mean age, $31.5 \pm$ 11.5 years.

youthful ciliary muscle, through to incipient and established presbyopia. Subjects with all types of refractive error were included, provided their distance prescription (including astigmatic component, if applicable) was amenable to correction with daily disposable soft contact lenses (Focus Dailies and Focus Dailies Toric: nelfilcon A, 69\% water content; Ciba Vision, Duluth, GA). The parameter ranges for these contact lenses meant subjects with spherical refractive errors greater than -10.00 DS or +6.00 DS were excluded from the investigation, as were those with oblique cylinders $>0.50 \mathrm{DC}$, or orthogonal cylinders $>1.50$ DC. The study was approved by the Ethics Committee of Aston University and was performed in accordance with the tenets of the Declaration of Helsinki. Written, informed consent was obtained from all participants after explanation of the nature and possible consequences of the study.

\section{Measurements}

Data were collected from the older cohort using the methods detailed previously. ${ }^{17}$ In brief, the process involved initial determination of refractive error in both eyes using an autorefractor (Grand Seiko Auto Ref/ Keratometer WAM-5500; Grand Seiko Co. Ltd., Hiroshima, Japan). ${ }^{25}$ Subjects with spherical or astigmatic error $>0.50 \mathrm{D}$ in either eye were corrected with disposable soft contact lenses (Focus Dailies or Focus Dailies Toric; Ciba Vision) to provide functional emmetropia at infinity, ensuring the accommodative demand was comparable for all participants. All further measurements were taken from the right eye only.

Objective accommodative responses to 4.0 and $8.0 \mathrm{D}$ accommodative stimuli (Maltese crosses) were determined with the WAM-5500, while the left eye was occluded with a patch. The targets subtended a constant angular subtense of $4.6^{\circ}$. Average target luminance and Michelson contrast values were $34.5 \mathrm{~cd} / \mathrm{m}^{2}$ and $82 \%$, and $30.5 \mathrm{~cd} / \mathrm{m}^{2}$ and $80 \%$, for the $4 \mathrm{D}$ and $8 \mathrm{D}$ stimuli, respectively. Many of the older participants were unable to attain/maintain clarity of either the 4.0 or 8.0 D targets, but were instructed to concentrate on the Maltese cross and make an effort to focus on $\mathrm{it}^{26}$ as blurred targets have been shown to stimulate ciliary muscle contraction, even in advanced presbyopes. ${ }^{11,13,14}$ Axial length was next determined from the mean of five readings obtained with an optical biometer (IOLMaster; Carl Zeiss Meditec. Inc., Dublin, CA). ${ }^{27}$ For those subjects corrected with soft contact lenses, axial length was measured once the lenses had been removed, after ciliary muscle image acquisition.

AS-OCT (Visante; Carl Zeiss Meditec, Inc.) ${ }^{28}$ images were obtained of nasal and temporal ciliary muscle in the relaxed state $(-0.19 \mathrm{D})$ and while viewing 4.0 and $8.0 \mathrm{D}$ accommodative stimuli. The scanning spot moves rapidly across the eye, acquiring $512 \mathrm{~A}$-scans in 0.25 seconds in high resolution mode, to generate a 2-dimensional image covering an area measuring $10 \mathrm{~mm}$ in width and $3 \mathrm{~mm}$ in depth. The scanning plane was set horizontally, at $0^{\circ}$, throughout the investigation. All participants were instructed to "carefully focus" on the accommodative stimuli during image acquisition, even if they were unable to maintain a clear image of the target. Maltese cross targets were used for fixation, positioned at an angle of $40^{\circ}$, such that the eccentric gaze of the subject, while their head was in the primary position on the chin and forehead rest, allowed images centered on the ciliary muscle to be captured. Forty degrees represented the minimum level of horizontal eye movement needed to view the distant targets, beyond the AS-OCT device, and meant that the optical axis of the instrument was through the sclera, rather than the cornea, reducing optical distortion. The distant target was viewed through a mirror, resulting in a stimulus vergence of $-0.19 \mathrm{D}$. Near targets, subtending $4.6^{\circ}$, were suspended in free space from an adjustable apparatus mounted on the AS-OCT headrest. Average luminance and Michelson contrast values were 38 $\mathrm{cd} / \mathrm{m}^{2}$ and $81 \%$, and $32 \mathrm{~cd} / \mathrm{m}^{2}$ and $78 \%$ for the $4 \mathrm{D}$ and $8 \mathrm{D}$ stimuli, respectively. Imaging was performed with the AS-OCT in high-resolution corneal mode throughout. For each of the two sides of the eye

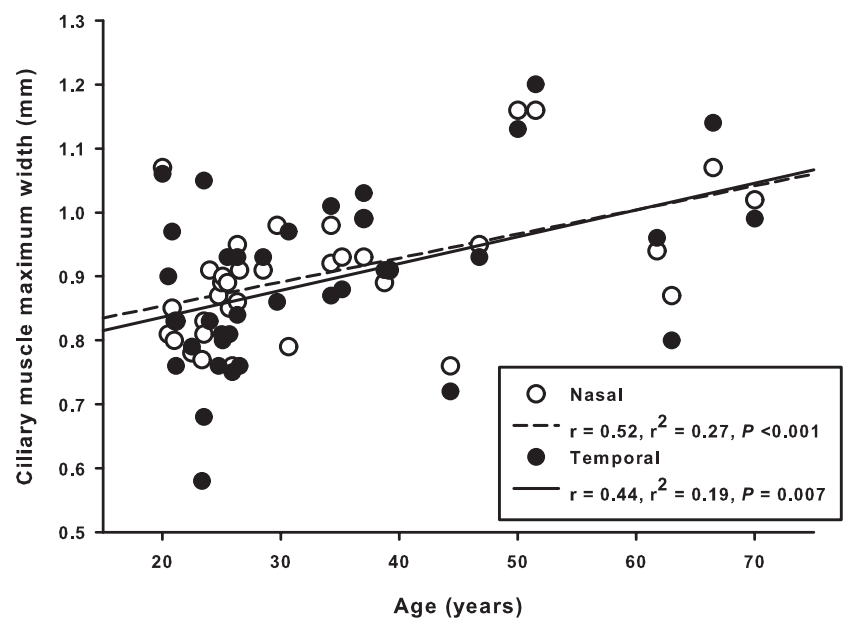

Figure 3. Nasal and temporal ciliary muscle maximum width versus age. $n=37$ participants. 


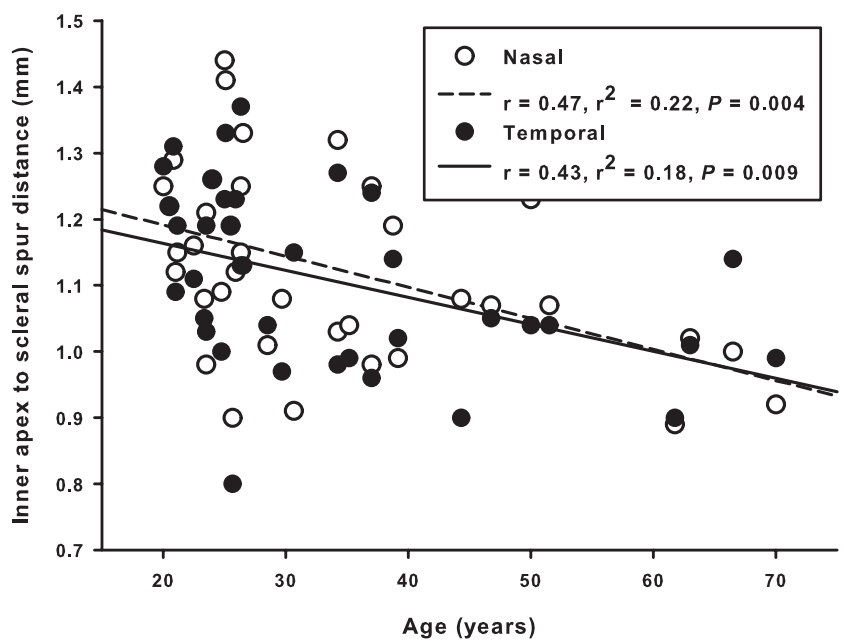

Figure 4. Nasal and temporal inner apex to scleral spur values versus age. $n=37$ subjects.

imaged, all targets were positioned along the same axis, with subjects asked to ensure that the near stimuli appeared directly over the distant Maltese cross to reduce the possibility of varying acquisition planes. Targets of the varying stimulus vergence levels were presented in random order, and multiple images were acquired of nasal and temporal ciliary muscle in each accommodative state, ensuring good visibility of the muscle in at least three images wherever possible.

\section{Image Analysis}

Image analysis was performed by one examiner (ALS) using the inbuilt software (Visante version 2.0; Carl Zeiss Meditec, Inc.). The examiner was masked to the accommodative state of subjects by use of a code to save image sets after scan acquisition. A refractive index of 1.000 was manually applied to all images before analysis. In addition to ciliary muscle length (the antero-posterior distance from the scleral spur) and thickness measures (width of the muscle at a point 25\% [CM25], 50\% [CM50], and 75\% [CM75] of the total length posterior to the scleral spur), ${ }^{17}$ two further parameters which may be relevant to the development of presbyopia, were determined from the AS-OCT images Ciliary muscle maximum thickness (Fig. 1) was measured using a caliper extending from the inner apex of the muscle to the muscle/ sclera boundary. As with all thickness measures detailed in the investigation, the caliper was positioned perpendicular to the muscle/ sclera interface. The distance from the scleral spur to the inner apex of the muscle (Fig. 2) was also determined to investigate the age-related shift in ciliary muscle location.

After analysis of the three nasal and temporal images in which the ciliary muscle was most clearly defined, using an applied refractive index of 1.000 , all width measures were divided by 1.382 , which is a more valid index for the ciliary muscle. ${ }^{29,30}$ Mean values for all length and width measures (adjusted for refractive index) were entered into a spreadsheet (Excel; Microsoft, Redmond, WA) and used for statistical analyses. The objective measures of accommodative response to the near stimuli were used to determine the magnitude of ciliary muscle biometric changes for each subject, per dioptre of accommodation.

Statistical Analysis. Linear regression analysis using graphing software (SigmaPlot Version 11; Systat Software Inc., Chicago, IL) was performed to determine the effect of age (independent variable) on nasal and temporal ciliary muscle morphologic characteristics. Mean and standard deviations were calculated if significant correlations were not found at the $\alpha=0.05$ level. Emmetropic and myopic eyes were considered separately in light of previous findings of altered ciliary muscle morphology in axial myopes. ${ }^{17}$ The few hyperopic participants were analyzed in conjunction with the emmetropes, as these eyes have not undergone myopic axial elongation.

\section{Results}

The total cohort of 79 subjects comprised 45 emmetropes/ hyperopes (mean spherical equivalent (MSE) $=-0.75$ to $+2.33 \mathrm{D}$ ) with a mean age of $34.7 \pm 12.8$ years, and 34 myopes (MSE $>-0.75 \mathrm{D}$ ), with a mean age of $31.5 \pm 11.5$ years. A wide range of refractive error was found among the whole cohort, ranging from $-9.50 \mathrm{D}$ to $+2.33 \mathrm{D} \mathrm{MSE}$, which was reflected by the broad spread of axial lengths, from 21.75 to $28.12 \mathrm{~mm}$ (mean $24.31 \pm 1.20 \mathrm{~mm}$ ). Mean refractive error and axial length values for the emmetropic participants (including the 6 hyperopes, with MSE $>+1.00 \mathrm{D})$ were $+0.31 \pm 0.70 \mathrm{D}$ and $23.68 \pm 0.83 \mathrm{~mm}$, respectively, and $-3.87 \pm 2.21 \mathrm{D}$ and $25.00 \pm 1.17 \mathrm{~mm}$, respectively, for the myopes. Table $1 \mathrm{sum}-$ marizes the effect of age on nasal and temporal ciliary muscle biometric characteristics.

\section{Ciliary Muscle Morphology}

Regarding ciliary muscle length measures, neither total length nor anterior length demonstrated a significant dependence on age in myopic eyes, for either the nasal (total length: $R=$ $0.325, P=0.065$; anterior length: $R=0.113, P=0.523$ ) or temporal (total length: $R=0.253, P=0.150$; anterior length: $R=0.183, P=0.300)$ aspects. In emmetropic eyes, ciliary muscle anterior length decreased significantly with age both nasally $(R=0.461, P=0.001)$ and temporally $(R=0.619, P<$ 0.001 ), while no age-related change in overall length was identified for either the nasal $(R=0.232, P=0.126)$ or temporal $(R=0.009, P=0.952)$ side.

Changes in thickness measures with age also displayed some refractive-group dependent asymmetry. CM2, measured at a fixed position $2 \mathrm{~mm}$ posterior to the scleral spur, did not demonstrate a significant age-related change in myopic eyes, either nasally $(R=0.119, P=0.504)$, or temporally $(R=$ $0.196, P=0.266$ ). However, in emmetropic eyes, CM2 re-

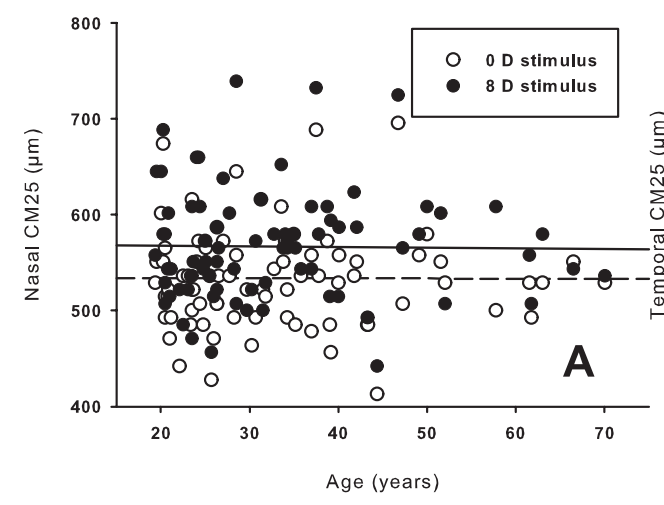

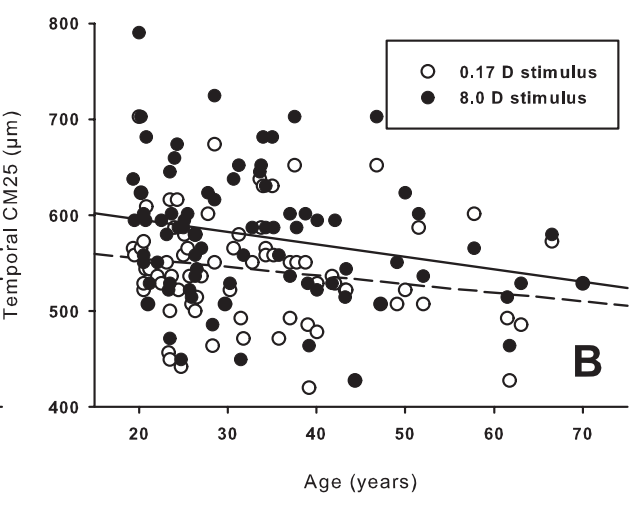

Figure 5. Nasal (A) and temporal (B) CM25 versus age for minimum accommodation (open circles and dashed regression line) and in response to an $8.0 \mathrm{D}$ stimulus (filled circles and solid regression line). $n=79$ subjects. 
Figure 6. Nasal (A) and temporal (B) anterior ciliary muscle length in emmetropes versus age for minimum accommodation (open circles and dashed regression line) and in response to an $8.0 \mathrm{D}$ stimulus (filled $n=45$ subjects. circles and solid regression line).
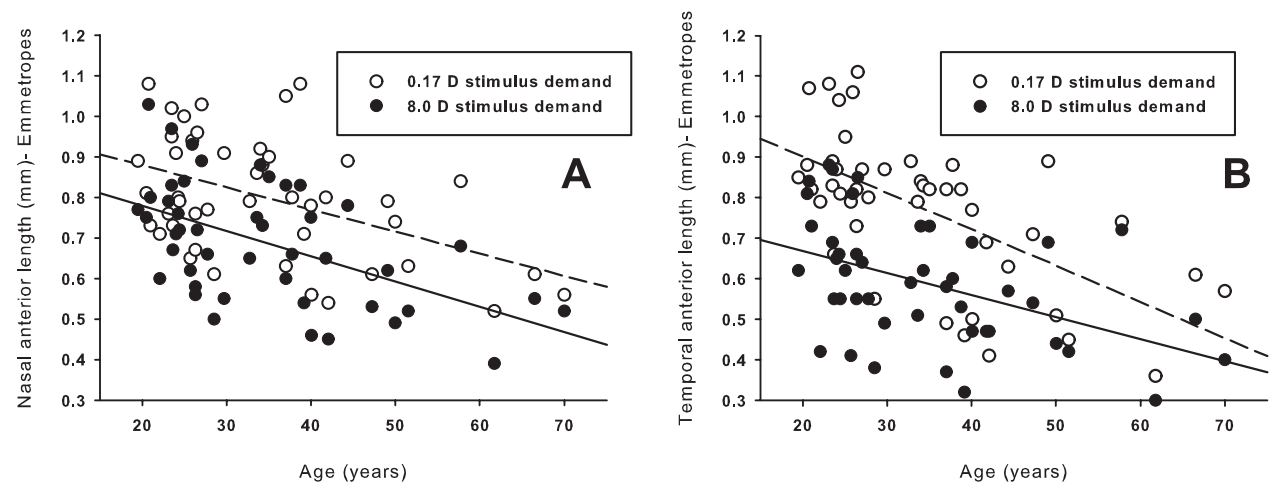

duced with age on the temporal side, by $2.08 \mu \mathrm{m} /$ year $(R=$ $0.387, P=0.009)$, but remained constant nasally $(R=0.193$, $P=0.009)$, with a mean value of $327 \pm 59 \mu \mathrm{m}$.

Regarding the proportional measures of ciliary muscle thickness, the most anterior of these parameters, CM25, did not show any dependence on age either nasally (emmetropes: $R=0.149, P=0.365$; myopes: $R=0.019, P=0.917$ ) or temporally (emmetropes: $R=0.009, P=0.956$; myopes: $R=$ $0.211, P=0.231$ ), for either refractive group. The more posterior CM50 and CM75 parameters showed an age dependent reduction in thickness on the temporal side for both refractive error groups [CM50 (emmetropes: $R=0.365, P=0.022$; myopes: $R=0.538, P=0.001$ ); CM75 (emmetropes: $R=$ $0.338, P=0.036$; myopes: $R=0.410, P=0.016$ )], although nasally, no significant age-related change was identified for either of these measures [CM50 (emmetropes: $R=0.076, P=$ 0.647; myopes: $R=0.129, P=0.469$ ); CM75 (emmetropes: $R=0.037, P=0.821$; myopes: $R=0.288, P=0.100)]$.

Ciliary muscle maximum width and distance from inner apex to scleral spur measures were obtained from only 37 of the 79 participants due to technical problems encountered with the AS-OCT device (hard-drive failure and unrecoverable image files). Despite the reduced data sets, the power of all statistical tests performed to ascertain the effect of age on these parameters was 0.80 or above. Ciliary muscle maximum width increased significantly with age, by $2.8 \mu \mathrm{m}$ / year nasally $(R=$ $0.54, P<0.001)$ and $3.0 \mu \mathrm{m} /$ year temporally $(R=0.44, P=$ $0.007)$. No significant difference between nasal and temporal maximum width measures was identified $(P=0.673)$. Figure 3 illustrates graphically the effect of age on maximum ciliary muscle width.

The distance from the inner apex of the ciliary muscle to the scleral spur decreased significantly with age on both the nasal and temporal aspects $(R=0.47 ; P=0.004$ and $R 0.43$; $P=0.009$, respectively). The reduction was similar on both sides: nasally, the decrease was $4.7 \mu \mathrm{m} /$ year, while on the temporal side, the change was $-4.1 \mu \mathrm{m} /$ year. No significant difference was identified between the nasal and temporal inner apex to scleral spur measures $(P=0.550)$. Figure 4 illustrates the relationship between age and ciliary muscle inner apex to scleral spur values.

\section{Ciliary Muscle Contractile Response}

CM25 (shown by Sheppard and Davies ${ }^{17}$ to increase significantly with accommodation in youthful eyes) thickens in response to an accommodative stimulus throughout life. Figure 5 illustrates graphically the change in nasal and temporal CM25 with accommodative effort versus age. Linear regression analysis revealed the magnitude of accommodative thickening of CM25 was not dependent on age for either the nasal or temporal aspects $\left(R=0.001, R^{2}=0.000, P=0.994\right.$ and $R=$ $0.202, R^{2}=0.041, P=0.090$, for nasal and temporal CM25, respectively).

In conjunction with the thickening of CM25, Sheppard and Davies $^{17}$ highlighted a shortening of ciliary muscle anterior length and overall length measures during accommodation. Both overall length and anterior length continue to reduce with accommodative effort throughout life. Figures 6 and 7 illustrate the change in nasal and temporal ciliary muscle anterior length in response to an $8.0 \mathrm{D}$ accommodative stimulus against subject age in emmetropes and myopes, respectively. In both refractive groups, the magnitude of change in anterior length during near vision is statistically unchanged with age on both the nasal $(R=0.254, P=0.161$ and $R=0.056, P=$ 0.755 , for emmetropes and myopes, respectively) and temporal $(R=0.061, P=0.741$ and $R=0.116, P=0.514$, for emmetropes and myopes, respectively) sides. Furthermore, the reduction in overall length during accommodative effort also remains statistically constant with age both nasally $(R=0.049$, $P=0.750$ and $R=0.073, P=0.680$, for emmetropes and myopes, respectively) and temporally $(R=0.075, P=0.624$ and $R=0.047, P=0.791$, for emmetropes and myopes, respectively). Figures 8 and 9 show the effect of age on nasal and temporal ciliary muscle overall length changes in response to an $8.0 \mathrm{D}$ accommodative stimulus.
Figure 7. Nasal (A) and temporal (B) ciliary muscle anterior length in myopes versus age for minimum accommodation (open circles and dashed regression line) and in response to an $8.0 \mathrm{D}$ stimulus (filled circles and solid regression line). $n=34$ subjects.
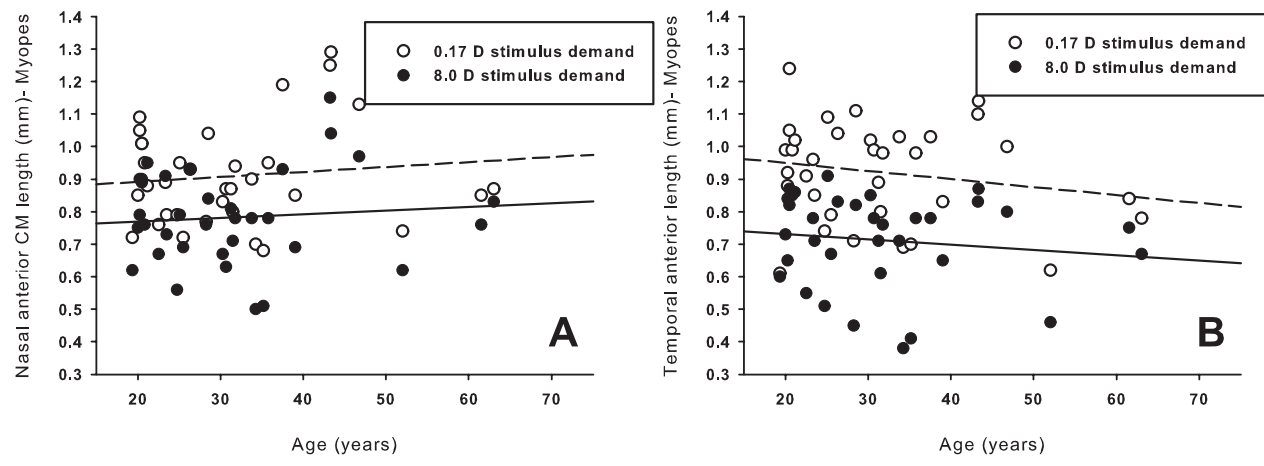


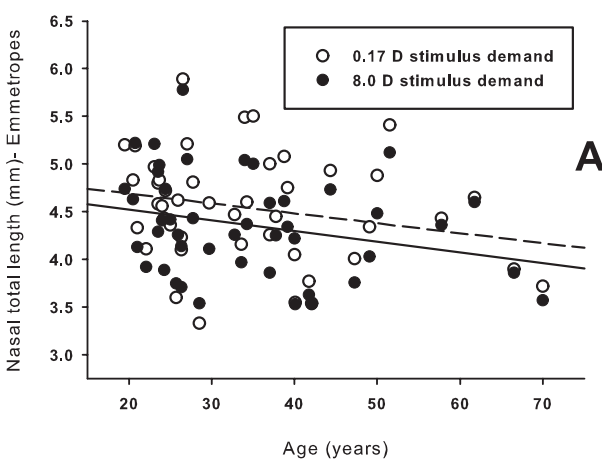

A

\section{Discussion}

There are limited published data documenting the effect of age on human ciliary muscle morphology and contractility. Previous in vitro and in vivo studies of ciliary muscle biometry have used narrower age-bands of subjects and/ or smaller cohort sizes. The present investigation represents the largest in vivo study to date to investigate relaxed and contracted ciliary muscle characteristics across a broad range of subject ages.

In the relaxed state, the anterior portion of the ciliary muscle becomes thicker throughout life, as reflected by the age-related increase in maximum width values, of approximately $3 \mu \mathrm{m}$ / year. More posteriorly, CM25 is unchanged in both refractive groups with age, while CM50 and CM75 become progressively thinner temporally. Regarding ciliary muscle lengths, the overall length is unchanged with age for both refractive groups, while anterior length measures decrease on both sides in emmetropic, but not myopic, eyes. Furthermore, the distance from the muscle inner apex to the scleral spur demonstrates a significant reduction with age, of approximately $4 \mu \mathrm{m} /$ year. Overall, the observed changes reflect a general antero-inwards shift of ciliary muscle mass throughout life. The anterior thickening of the ciliary muscle is in agreement with a recent in vivo MRI study ${ }^{11}$ and earlier in vitro data. ${ }^{6}$ Increased thickness of the anterior portion of the muscle may be a consequence of the age-related build-up of connective tissue observed in human post mortem samples, ${ }^{6}$ particularly in the radial and circular regions, ${ }^{12,31}$ and/ or a change in the relative proportions of different fiber orientations. The quantity of circular ${ }^{31}$ and radial $^{6}$ fibers, compared to the longitudinal portion, may increase with age, resulting in a bulging of the anterior portion of the ciliary muscle.

Thinning of the posterior region of the human ciliary muscle has not previously been documented, although the present study has identified a significant reduction in temporal CM50 and CM75 in both refractive groups. Sheppard and Davies ${ }^{17}$ highlighted increased thickness of CM50 and CM75 parameters on the temporal side, compared to the nasal aspect, in youthful eyes. The age-dependent decrease in posterior thickness may be a consequence of a reduction in longitudinally orientated muscle fibers, compared to radial and circular fibers, on the temporal side. Tamm et al. ${ }^{12}$ measured an in vitro decrease in the area of the longitudinal portion of the muscle in older eyes, but did not state from which region of the globe (i.e., nasal/ temporal/ superior/ inferior) the samples were obtained.

Hitherto, age-dependent changes in ciliary muscle length and distance from the inner apex to scleral spur have been observed in several in vitro investigations, but not previously reported in vivo. The reduction in distance from inner apex to scleral spur is in accordance with the in vitro data of Tamm et al. ${ }^{12}$ from donor eyes aged 33-87 years. No other previously published study has investigated the change in this parameter in the human eye with age. Ciliary muscle length changes with age have been more widely investigated, with some disagreement between studies. Tamm et al. ${ }^{12}$ documented a continuous and significant decline in overall length with age, whereas Pardue and Sivak ${ }^{6}$ found no statistically significant change in this parameter, although the length of the anterior portion showed a substantial reduction. The present study has identified no significant age-dependent change in ciliary muscle total length with age, in either emmetropes or myopes, although the anterior length reduced significantly in the emmetropic group. Sheppard and Davies ${ }^{17}$ identified a positive correlation between axial length and both anterior and overall length measures, suggesting that the ciliary muscle elongates during myopigenesis. The maintenance of a constant anterior muscle length with age in myopic subjects suggests that the forces responsible for elongation of the globe are still present in later life, and inhibit the anterior shift of ciliary muscle mass that occurs in emmetropes.

The mechanism responsible for the age-related anterior shift in ciliary muscle mass is unclear, although previous authors have provided hypotheses relating to this matter. According to the Modified Geometric Theory of presbyopia development, postulated by Strenk et al., ${ }^{1}$ growth of the lens results in antero-inwards movement of the uveal tract, through forces transmitted via the pupillary margin to the iris root. As the lens
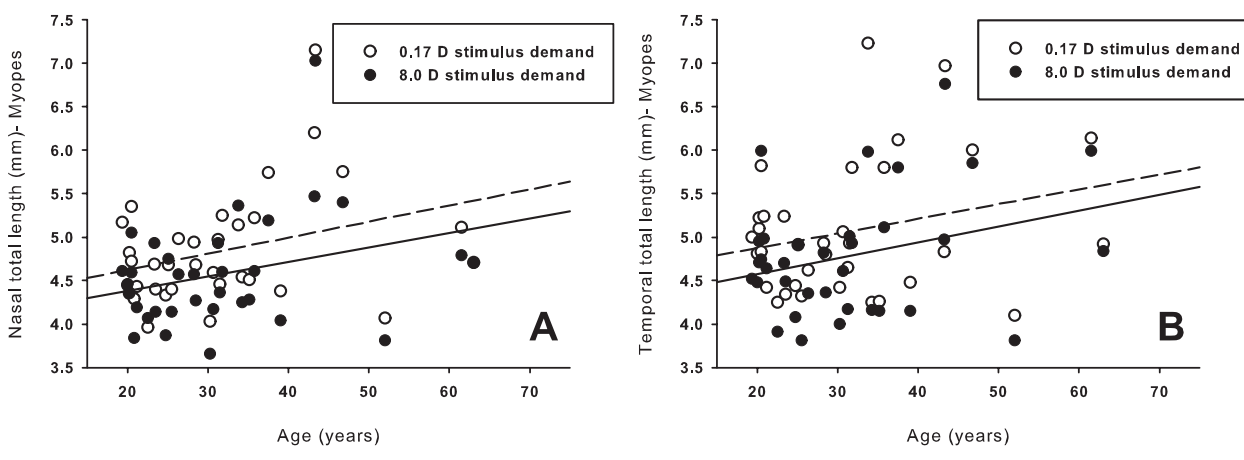

Figure 9. Nasal (A) and temporal (B) ciliary muscle total length in myopes versus age for minimum accommodation (open circles and dashed regression line) and in response to an $8.0 \mathrm{D}$ stimulus (filled circles and solid regression line). $n=34$ subjects. 
becomes progressively thicker throughout life, ever-increasing force is applied to the pupillary margin (which is in contact with the anterior lens surface) and translated to the iris root and ciliary muscle. In addition to an anterior movement of the ciliary muscle, an inwards displacement simultaneously occurs, due to the constraining effect of the curved sclera. The anteroinwards movement of the uvea leads to reduced pupil diameter, causing the pupillary margin to become increasingly nearer to the thicker part of the lens, and augmenting the forces applied by the lens. An alternative possible explanation for age-related anterior and inwards displacement of the ciliary muscle is based on the migration of zonules over the surface of the lens throughout life. Pardue and Sivak ${ }^{6}$ hypothesized that the forward migration of zonular insertions into the lens capsule $^{32,33}$ due to lens thickening with advancing age, causes an anterior and inwards pull on the ciliary body.

Regarding ciliary muscle function in the ageing eye, the results of the present study suggest that there is no significant decrease in the contractile ability of the muscle, even in eyes with established presbyopia. The main accommodative changes in ciliary muscle morphology observed in Sheppard and Davies ${ }^{17}$ were a reduction in overall length and anterior length measures, with a concurrent thickening of CM25. Figures 5 to 9 inclusive indicate that these parameters show the same changes in response to a near stimulus throughout life: linear regression analysis of the change in each measure between the minimal and 8.0 D stimulus levels versus age showed no statistical dependence of the magnitude of change on subject age. The data support a lensocentric model of presbyopia development, whereby continued growth and alterations in lenticular viscoelastic properties reduce the ability of the capsule to mold the lens into an accommodated form. Furthermore, the anterior migration of zonular insertions over the enlarged lens, such that they become more tangential to the lenticular surface with advancing age, may act to reduce the ability of the zonules to impart tension on the capsule.

In considering the findings of the current investigation, attention must be paid to the limitations of the study. While the technique used allowed relatively high resolution and rapid imaging of the ciliary muscle, AS-OCT has a number of associated drawbacks. The contrast and field of view of the images acquired are limited, compared to MRI, meaning it is not possible to image both the nasal and temporal ciliary muscle simultaneously. In common with several imaging techniques, AS-OCT is affected by optical distortion and relies on correction algorithms to dewarp the images; these were removed in the present study and a refractive index of 1.382 applied for the ciliary muscle. Furthermore, the technical problems encountered with the AS-OCT meant it was not possible to obtain ciliary muscle maximum width and inner apex to scleral spur measures from all participants. Due to the reduced data set, it was not feasible to compare the age-related changes in these parameters in emmetropes versus hyperopes. While inner apex to scleral spur measures decreased with age (in $n=37$ participants, mixed refractive errors), suggesting an anterior displacement of the ciliary muscle with age, the anterior length was found to significantly decrease only in emmetropic eyes. It is possible that refractive group differences exist in the agerelated changes in inner apex to scleral spur and maximum thickness measures, which were not detected in the present study. Additional further investigation could serve to clarify this matter.

Thus, the human ciliary muscle undergoes age-dependent changes in morphology that suggest an antero-inwards displacement of muscle mass, particularly in emmetropic eyes. The morphologic changes observed appear not to affect the ability of the muscle to contract, even in established presby- opes, thus supporting a lenticular model of presbyopia development.

\section{References}

1. Strenk SA, Strenk LM, Koretz JF. The mechanism of presbyopia. Prog Retin Eye Res. 2005;24:379-393.

2. Charman WN. The eye in focus: accommodation and presbyopia. Clin Exp Optom. 2008;91:207-225.

3. Glasser A. Restoration of accommodation: surgical options for correction of presbyopia. Clin Exp Optom. 2008;91:279-295.

4. Ziebarth NM, Borja D, Arrieta E, et al. Role of the lens capsule on the mechanical accommodative response in a lens stretcher. Invest Ophthalmol Vis Sci. 2008;49:4490-4496.

5. Truscott RJ. Presbyopia. Emerging from a blur towards and understanding of the molecular basis for this most common eye condition. Exp Eye Res. 2009;88:241-247.

6. Pardue MT, Sivak JG. Age-related changes in human ciliary muscle. Optom Vis Sci. 2000;77:204-210.

7. Tamm E, Lütjen-Drecoll E, Jungkunz W, Rohen JW. Posterior attachment of ciliary muscle in young, accommodating old, presbyopic monkeys. Invest Ophthalmol Vis Sci. 1991;32:1678-1692.

8. Tamm E, Croft MA, Jungkunz W, Lütjen-Drecoll E, Kaufman PL. Age-related loss of ciliary muscle mobility in the rhesus monkey: role of the choroid. Arch Opbthalmol. 1992;110:871-876.

9. Harocopos GJ, Shui YB, McKinnon M, Holekamp NM, Gordon MO, Beebe DC. Importance of vitreous liquefaction in age-related cataract. Invest Ophthalmol Vis Sci. 2004;45:77-85.

10. Park KH, Yun JH, Kee C. The effect of cataract extraction on the contractility of ciliary muscle. Am J Ophthalmol. 2008;146:8-14.

11. Strenk SA, Strenk LM, Guo S. Magnetic resonance imaging of the anteroposterior position and thickness of the aging, accommodating, phakic and pseudophakic ciliary muscle. J Cataract Refract Surg. 2010;36:235-241.

12. Tamm S, Tamm E, Rohen JW. Age-related changes of the human ciliary muscle: a quantitative morphometric study. Mech Ageing Dev. 1992;62:209-221.

13. Strenk SA, Semmlow JL, Strenk LM, Munoz P, Gronlund-Jacob J, De Marco JK. Age-related changes in human ciliary muscle and lens: a magnetic resonance imaging study. Invest Opbthalmol Vis Sci. 1999; 40:1162-1169.

14. Strenk SA, Strenk LM, Guo S. Magnetic resonance imaging of aging, accommodating, phakic, and pseudophakic ciliary muscle diameters. J Cataract Refract Surg. 2006;32:1792-1798.

15. Glasser A, Croft MA, Brumback L, Kaufman PL. Ultrasound biomicroscopy of the aging rhesus monkey ciliary region. Optom Vis Sci. 2001;78:417-424.

16. Croft MA, Glasser A, Heatley G, et al. The zonula, lens, and circumlental space in the normal iridectomized rhesus monkey eye. Invest Ophthalmol Vis Sci. 2006;47:1087-1095.

17. Sheppard AL, Davies LN. In vivo analysis of ciliary muscle morphologic changes with accommodation and axial ametropia. Invest Opbthalmol Vis Sci. 2010;51:6882-6889.

18. von Helmhotz HH. Handbuch der Physiologischen Optik. New York: Dover Publications; 1909.

19. Croft MA, Glasser A, Kaufman PL. Accommodation and presbyopia. Int Opbthalmol Clinics. 2001;41:33-46.

20. Koeppl C, Findl O, Kriechbaum K, Drexler W. Comparison of pilocarpine-induced and stimulus-driven accommodation in phakic eyes. Exp Eye Res. 2005;80:795-800.

21. Kriechbaum K, Findl O, Koeppl C, Menapace R, Drexler W. Stimulus-driven versus pilocarpine-induced biometric changes in pseudophakic eyes. Ophthalmology. 2005;112:453-459.

22. Uthoff D, Holland D, Hepper D, Gulati A, Koch L, Haigis W. Laserinterferometric measurements of accommodative changes in the position of an optic-shift intraocular lens. J Refract Surg. 2009;25:416-420.

23. Bailey MD, Sinnott LT, Mutti DO. Ciliary body thickness and refractive error in children. Invest Opbthalmol Vis Sci. 2008;49: 4353-4360.

24. Nubile M, Calienno R, Lanzini M, Mastropasqua L. Applications for Visante OCT. Cataract and Refractive Surgery Today Europe. 
2008. Available online at http://www.crstodayeurope.com/Pages/ whichArticle.php?id=323. Accessed February 9, 2010.

25. Sheppard AL, Davies LN. Clinical Evaluation of the Grand Seiko Auto Ref/ Keratometer WAM-5500. Ophthalmic Physiol Opt. 2010 30:143-151

26. Stark LR, Atchison DA. Subject instructions and methods of target presentation in accommodation research. Invest Ophthalmol Vis Sci. 1994;35:528-537.

27. Santodomingo-Rubido J, Mallen EAH, Gilmartin B, Wolffsohn JS. A new non-contact device for ocular biometry. Br J Ophthalmol. 2002;86:458-462.

28. Dunne MCM, Davies LN, Wolffsohn JS. Accuracy of cornea and lens biometry using anterior segment optical coherence tomography. J Biomed Opt. 2007;12(6):064023.
29. Tearney GJ, Brezinski ME, Southern JF, Bouma BE, Hee MR, Fujimoto JG. Determination of the refractive index of highly scattering human tissue by optical coherence tomography. Opt Lett. 1995;20:2258-2261.

30. Dirckx JJ, Kuypers LC, Decraemer WF. Refractive index of tissue measured with confocal microscopy. J Biomed Opt. 2005;10(4):044014.

31. Nishida S, Mizutani S. Quantitative and morphometric studies of age-related changes in human ciliary muscle. Jpn J Ophthalmol. 1992;36:380 -387.

32. Farnsworth PN, Shyne SE. Anterior zonular shifts with age. Exp Eye Res. 1979;28:291-297.

33. Sakabe I, Oshika T, Lim SJ, Apple DJ. Anterior shift of zonular insertion into the anterior surface of human crystalline lens with age. Ophthalmology. 1998;105:295-299. 